\title{
Induction of mesenchymal cell phenotypes in lung epithelial cells by adenovirus E1A
}

\author{
A.R. Behzad, K. Morimoto, J. Gosselink, J. Green, J.C. Hogg and S. Hayashi
}

\begin{abstract}
Epithelial-mesenchymal transformation is now recognised as an important feature of tissue remodelling. The present report concerns the role of adenovirus infection in inducing this transformation in an animal model of chronic obstructive pulmonary disease.
\end{abstract}

Guinea pig primary peripheral lung epithelial cells (PLECs) transfected with adenovirus E1A (E1A-PLECs) were compared to guinea pig normal lung fibroblasts (NLFs) transfected with E1A (E1A-NLFs). These cells were characterised by PCR, immunocytochemistry, electron microscopy, and Western and Northern blot analyses. Electrophoretic mobility shift assays were performed in order to examine nuclear factor (NF)-KB and activator protein (AP)-1 binding activities.

E1A-PLECs and E1A-NLFs positive for E1A DNA, mRNA and protein expressed cytokeratin and vimentin but not smooth muscle $\alpha$-actin. Both exhibited cuboidal morphology and junctional complexes, but did not contain lamellar bodies or express surfactant protein A, B or C mRNAs. These two cell types differed, however, in their NF-KB and AP-1 binding after lipopolysaccharide stimulation, possibly due to differences in the expression of the subunits that comprise these transcriptional complexes.

E1A transfection results in the transformation of peripheral lung epithelial cells and normal lung fibroblasts to a phenotype intermediate between that of the two primary cells. It is postulated that this intermediate phenotype may play a major role in the remodelling of the airways in chronic obstructive pulmonary disease associated with persistence of adenovirus E1A DNA.

KEYWORDS: Adenovirus E1A, alveolar type-Il cell, epithelial-mesenchymal transformation, lung fibroblasts, pathogenesis of chronic obstructive pulmonary disease, transcription factors

hronic obstructive pulmonary disease (COPD) is a major cause of morbidity and mortality worldwide [1]. The loss of lung elastic recoil caused by emphysema and the remodelling process that obstructs the small airways cause the gradual decline in forced expiratory volume in one second that characterises this condition [2]. Recent studies based on the National Institutes of Health-sponsored National Emphysema Treatment Trial have shown that the progression of COPD is associated with thickening of the walls of the small conducting airways by a repair and remodelling process [2]. Although inhalation of toxic gases and particles is the major risk factor for COPD [3], viral infections, particularly childhood infection, add to the risk of developing this condition [1]. Adenoviral DNA is retained by peripheral epithelial cells of the lung (PLECs) in humans in whom the viral E1A gene is expressed [4] without replication of a complete virus [5]. Case-control studies have shown that the E1A DNA from group $\mathrm{C}$ adenoviruses is present in greater amounts in the lungs of patients with COPD than in controls with normal lung function matched for age, sex and smoking history [5], and that the excess inflammation in the lungs of smokers with severe emphysema is associated with increased numbers of alveolar epithelial cells expressing E1A [6].

These human observations are supported by experiments in guinea pigs, where, in the absence of actively replicating virus but the presence of E1A DNA, adenovirus infection resulted in excess lung inflammation following a single exposure to cigarette smoke [7], and increased parenchymal and airway wall inflammation with greater emphysematous destruction after chronic exposure [8]. Furthermore, E1A DNA and protein persisted in epithelial cells in the lungs of these animals [9].

Reports from several laboratories suggest that a phenomenon termed epithelial-mesenchymal transformation, in which epithelial cells transform into fibroblasts capable of producing connective tissue matrix, is an important feature of tissue remodelling $[10,11]$. In the present study,
AFFILIATIONS

James Hogg iCapture Center for Cardiovascular and Pulmonary Research, St Paul's Hospital, Vancouver, BC, Canada.

\section{CORRESPONDENCE}

S. Hayashi

University of British Columbia James Hogg iCapture Center for

Cardiovascular and Pulmonary

Research

St Paul's Hospital

1081 Burrard Street

Vancouver

$\mathrm{BC}$

V6Z 1 Y 6

Canada

Fax: 16048068351

E-mail: shayashi@mrl.ubc.ca

Received:

May 202005

Accepted after revision:

July 312006

SUPPORT STATEMENT

This study was supported by Canadian Institutes of Health Research (Ottawa, ON, Canada) grant No. 7246. 
the hypothesis that adenoviral infection followed by persistent expression of its E1A gene is capable of producing epithelialmesenchymal transformation was examined by investigating guinea pig PLECs that express adenoviral E1A. The changes that occur during adenoviral E1A transformation of primary guinea pig PLECs were also compared with those occurring during the transformation of the fibroblasts that frequently contaminate primary epithelial cultures, and evidence is presented that both cell types are transformed to an intermediate phenotype.

\section{MATERIALS AND METHODS \\ Animals}

Female pathogen-free guinea pigs (Cavia porcellus; Cam Hartley strain; 200-250 g) were obtained from Charles River Canada (St-Constant, QC, Canada). These studies were approved by the Committee on Animal Care of the University of British Columbia (Vancouver, BC, Canada).

\section{Isolation and culture of PLECs}

PLECs were isolated from guinea pig lungs as described previously [12], with one modification to improve purity. Instead of immunoglobulin ( $\mathrm{Ig}) \mathrm{G}$ panning, panning crude cell suspensions on uncoated $75 \mathrm{~cm}^{2}$ culture flasks for $30 \mathrm{~min}$ and collecting nonadherent cells provided $3.0 \pm 0.5 \times 10^{7}$ PLECs per guinea pig $(n=14)$, with $93 \pm 5 \%$ of cells excluding trypan blue dye.

Purified PLECs were plated at $3 \times 10^{5}-5 \times 10^{5}$ cells $\cdot \mathrm{cm}^{-2}$ on uncoated six-well plates or 24-mm Transwell ${ }^{\mathrm{TM}}$ plates (Costar, Cambridge, MA, USA) containing tissue culture-treated polycarbonate (nucleopore) inserts coated with $1 \mathrm{~mL} \mathrm{2:1} \mathrm{(volume}$ (v)/v) Matrigel (Collaborative Biomedical Products, Bedford, MA, USA)/Dulbecco's modified Eagle medium (DMEM; Gibco BRL, Inc., Gaithersburg, MD, USA) and cultured in DMEM containing 10\% foetal bovine serum (FBS; HyClone Laboratories, Logan, UT, USA), 1\% penicillin/streptomycin (Sigma Chemical Co., St Louis, MO, USA), $10 \mu \mathrm{g} \cdot \mathrm{mL}^{-1}$ gentamicin (Gibco BRL, Inc.) and $2.5 \mu \mathrm{g} \cdot \mathrm{mL}^{-1}$ Fungizone (Gibco BRL, Inc.). On the following day, adherent cells were fed with DMEM containing $5 \mathrm{ng} \cdot \mathrm{mL}^{-1}$ keratinocyte growth factor (Sigma Chemical Co.) to stimulate type-II cell proliferation [13]; penicillin/streptomycin, gentamicin and Fungizone as above; $2 \%$ FBS; $1 \times$ insulin-transferrin-selenium (Sigma Chemical Co.); $10 \mathrm{ng} \cdot \mathrm{mL}^{-1}$ epidermal growth factor (Sigma Chemical Co.) and $100 \mathrm{nM}$ hydrocortisone (Sigma Chemical Co.).

\section{Assessment of cell purity in primary culture}

PLECs from two guinea pigs were separately purified and plated in culture dishes containing three glass coverslips. The cells from one guinea pig were analysed after 3 days and those from the other after 4 days of culture. Following cold methanol fixation, cells were examined using light microscopy. The

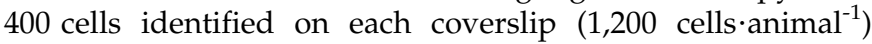
were classified as either PLECs (cobblestone morphology), normal lung fibroblasts (NLFs; spindle-shaped morphology) or unclassified.

\section{Immortalisation of primary PLECs}

The plasmid pE1Aneo (from F. Graham, McMaster University, Hamilton, ON, Canada) carries the entire E1A gene with its promoter (nucleotides 25-1770 of adenovirus 5) and the neomycin resistance gene, driven by the simian virus 40 large T-antigen promoter.

Primary PLECs from eight guinea pigs were cultured at $3 \times 10^{5}-5 \times 10^{5}$ cells $\cdot \mathrm{cm}^{-2}$ on uncoated six-well plates or in Matrigel-coated Transwell plates for 1-4 days before exposure to $\mathrm{pE} 1$ Aneo complexed to Lipofectamine reagent (Gibco BRL, Inc.) for $6 \mathrm{~h}$ according to the manufacturer's directions. After 3 days, cells were selected (for neomycin resistance) with $250 \mu \mathrm{g} \cdot \mathrm{mL}^{-1} \mathrm{G} 418$ (Gibco BRL, Inc.), an aminoglycoside. Cells grown on uncoated six-well plates that were resistant after 2 weeks were replated on to new six-well plates, whereas those grown on Matrigel were first harvested as described previously [14] and then re-plated on to uncoated six-well plates. After selection of G418-resistant single-cell clones, all subsequent analysis of a selected number of these clones (see Results section) was carried out on cells from passages three to four. The same restriction on passage number was applied to the E1A-NLFs below.

\section{Culture and immortalisation of guinea pig NLFs}

NLFs, isolated as described previously [15] from two guinea pigs, were plated on to separate uncoated six-well plates at a density of $2 \times 10^{4}$ cells $\cdot \mathrm{cm}^{-2}$ in DMEM containing $10 \%$ FBS and $1 \%$ penicillin/streptomycin. Cells were cultured for 2-3 days before transfection with the pE1Aneo-liposome complex. G418-resistant cells were re-plated on 12-well plates.

\section{Detection of E1A DNA, MRNA and protein}

E1A DNA in DNA extracted from transfected and untransfected cells was determined by PCR as described previously [7]. RNA from these cells was reverse transcribed and then amplified using forward and reverse primers 5'-AGTGACGACGAGGATGAAGAGG-3' and 5'-TCTCAGGAGGTGTGTTAGAAGG-3', equivalent to nucleotides 953-974 and 1381-1402, respectively, for the adenovirus type 5 sequence (Genbank gi: 58484). The predicted sizes of the PCR products were 194 and 334 base pairs for the 12 and 13S E1A mRNAs, respectively. E1A protein was detected by immunofluorescence staining [4] and Western blotting.

\section{Characterisation of primary and transfected cells}

\section{Electron microscopy}

Freshly isolated PLECs $\left(\sim 3.0 \times 10^{7}\right)$ were processed into five Epon blocks for transmission electron microscopy [16]. One ultrathin section from each block was stained with uranyl acetate and lead citrate for examination using a Philips 400 transmission electron microscope (Philips, Eindhoven, the Netherlands). The number of each cell type was expressed as a percentage of the total number of cells examined. The morphology of these cells was compared to that of purified PLECs and PLEC transfectants cultured on polycarbonate inserts coated with 2:1 (v/v) Matrigel/DMEM in 24-mm Transwell plates for 4 days. Untransfected and transfected NLFs, confluent after culture for 4 days on uncoated polycarbonate inserts inside 24-mm Transwell plates, were examined in a similar manner.

Immunohistochemistry

Immunohistochemistry [17] was performed using mouse monoclonal antihuman antibodies (Dako, Glostrup, Denmark) directed against cytokeratins (acidic and basic) and vimentin at 
$4.6 \mu \mathrm{g} \cdot \mathrm{mL}^{-1}$ and smooth muscle $\alpha$-actin at $5.0 \mu \mathrm{g} \cdot \mathrm{mL}^{-1}$. Respective concentrations of an isotype-matched mouse IgG (Sigma Chemical Co.) served as controls. The staining intensity of 30 cells in random fields of view was scored as - (identical to negative control),+ (moderate staining) or ++ (intense staining) by visual assessment.

Northern blot analysis of surfactant protein mRNAs

RNA was extracted from freshly purified PLECs or primary PLECs cultured on $100-\mathrm{mm}$ plastic dishes or polycarbonate inserts coated with Matrigel inside 24-mm Transwell plates and the E1A transfectants using TRIzol (Gibco BRL, Inc.), whereas RNeasy Maxi kits (Qiagen, Inc., Mississauga, ON, Canada) were used for guinea pig lung. Northern blot analysis followed, using the complementary DNAs listed below.

Human surfactant protein A, B and C and 18S ribosomal complementary DNA probes cross-hybridise with the respective guinea pig RNAs. Probes were labelled with $\left[\alpha_{-}{ }^{32} \mathrm{P}\right]$ deoxycytidine triphosphate (Amersham, Piscataway, NJ, USA) by random priming.

\section{Electrophoretic mobility shift assay}

Cells grown on $75 \mathrm{~cm}^{2}$ flasks were stimulated with $10 \mu \mathrm{g} \cdot \mathrm{mL}^{-1}$ lipopolysaccharide (LPS) for $2 \mathrm{~h}$. Nuclear extracts were prepared from these and untreated cells, and electrophoretic mobility shift assays (EMSAs) for nuclear factor (NF)- $\kappa \mathrm{B}$ and activator protein (AP)-1, as well as the respective cold competition and supershift assays, were performed as previously described [18].

\section{RESULTS}

\section{Assessment of primary PLEC purity}

Light microscopic examination of 1,200 primary PLECs cultured for 3 days showed that $50.3 \pm 6.7 \%$ exhibited cobblestone morphology typical of PLECs, $4.6 \pm 0.7 \%$ were spindle-shaped fibroblasts and $45 \pm 7.3 \%$ rounded cells of undetermined origin. Another 1,200 cells examined after 4 days of culture were $45.8 \pm 6.9 \%$ PLECs, $15.8 \pm 1.7 \%$ fibroblasts and $38.4 \pm 5.3 \%$ of undetermined origin.

\section{Immortalisation of primary PLECs and NLFs}

Primary PLECs grown on uncoated plates and transfected with pE1Aneo produced G418-resistant colonies in three of the 12 wells. Nine resistant single-cell clones (E1A-PLEC clones 1-9) were expanded from one of these colonies and all nine were maintained under constant selection with $100 \mu \mathrm{g} \cdot \mathrm{mL}^{-1} \mathrm{G} 418$. Similarly, primary PLECs grown on Matrigel produced G418resistant colonies in two of the 12 wells, from which six resistant single-cell clones (E1A-PLEC clones 10-15) were established. Cells from all 15 PLEC clones exhibited cobblestone morphology and continued to divide after reaching confluence. Clones 1, 2, 10 and 11 were used in the present study.

Primary NLFs transfected with pE1Aneo produced G418resistant colonies in four of the 12 wells. One clone from a single resistant cell was obtained from each (E1A-NLF clones 1-4). All four showed cobblestone morphology, continued to divide after reaching confluence and were maintained under selection with G418. E1A-NLF clones 1 and 2 were used in the present study.
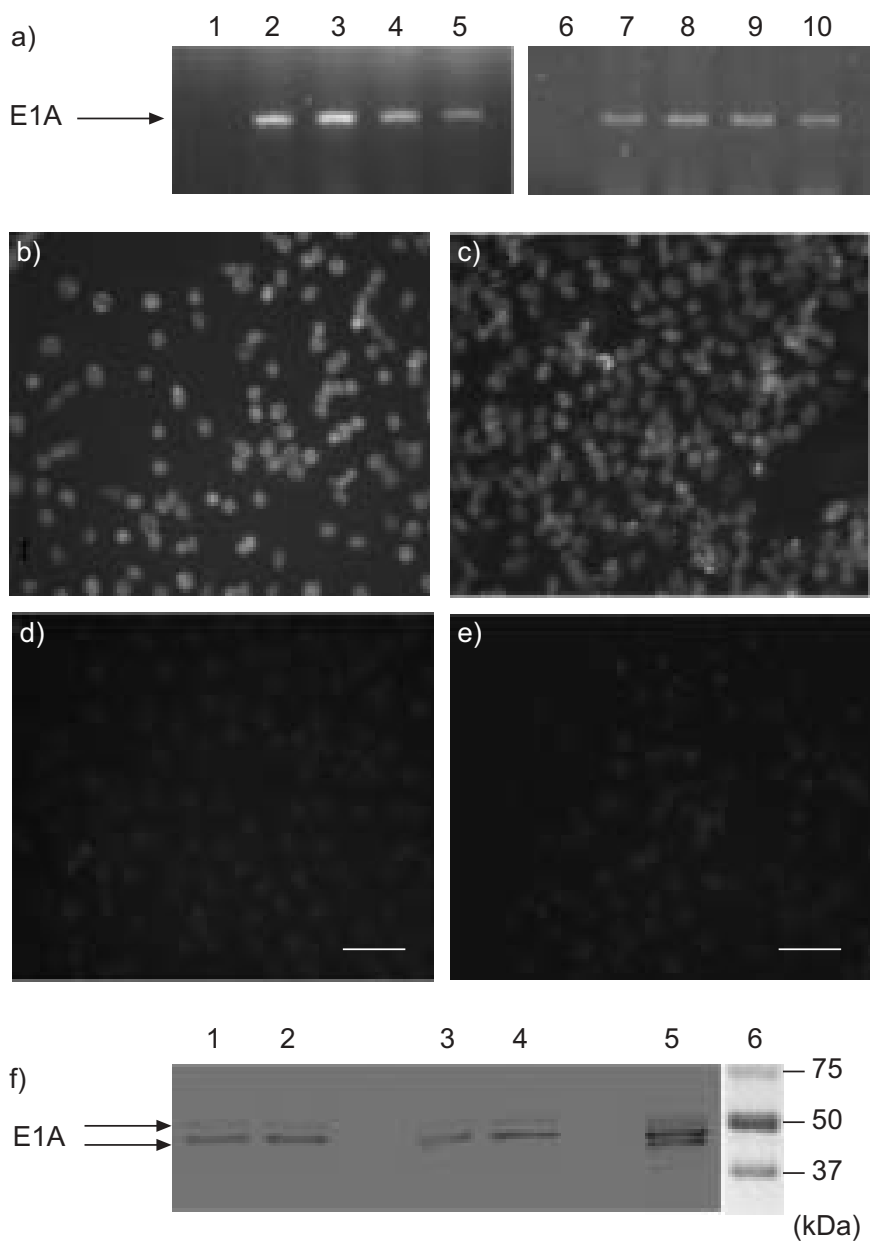

g)

$(\mathrm{kDa})$

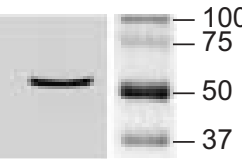

$(\mathrm{kDa})$

h)

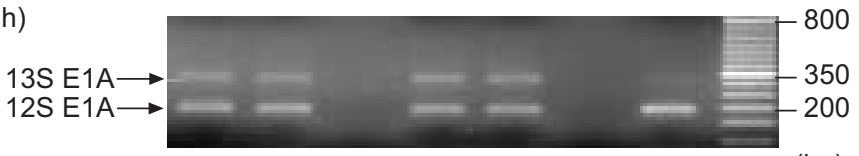

(bp)

FIGURE 1. Detection of adenovirus E1A DNA by PCR, mRNA by reverse transcriptase (RT)-PCR, and protein by immunofluorescence staining and Western blotting. a) Ethidium-bromide-stained agarose gels of E1A PCR products (486 base pairs (bp)) from E1A peripheral lung epithelial cell (PLEC) clones 1, 2, 10 and 11 (lanes 2-5, respectively) and E1A normal lung fibroblast (NLF) clones 1-4 (lanes 710, respectively). Untransfected PLECs (lane 1) and untransfected NLFs (lane 6) were used as negative controls. Monoclonal antibody directed against E1A (b, c) and isotypic control antibody (d, e) were applied to E1A-PLEC clone 1 (b, d) and E1A-NLF clone 1 (c, e) Scale bars $=10 \mu \mathrm{m}$. Application of the same E1A antibody to a Western blot (f) revealed two E1A isoforms in E1A-PLEC clones 1 and 2 (lanes 1 and 2) and E1A-NLF clones 1 and 2 (lanes 3 and 4) that were equivalent to two of the largest E1A polypeptides detected in G293 cells (lane 5). An antibody directed against $\beta$-tubulin was used to control for protein loading in the corresponding lanes (g). The corresponding size markers are shown in lane 6. h) Ethidium bromidestained agarose gel of RT-PCR products of E1A mRNA (lanes as in f), except adenovirus 5-infected A549 cells replaced G293 cells as a positive control in lane 5). 

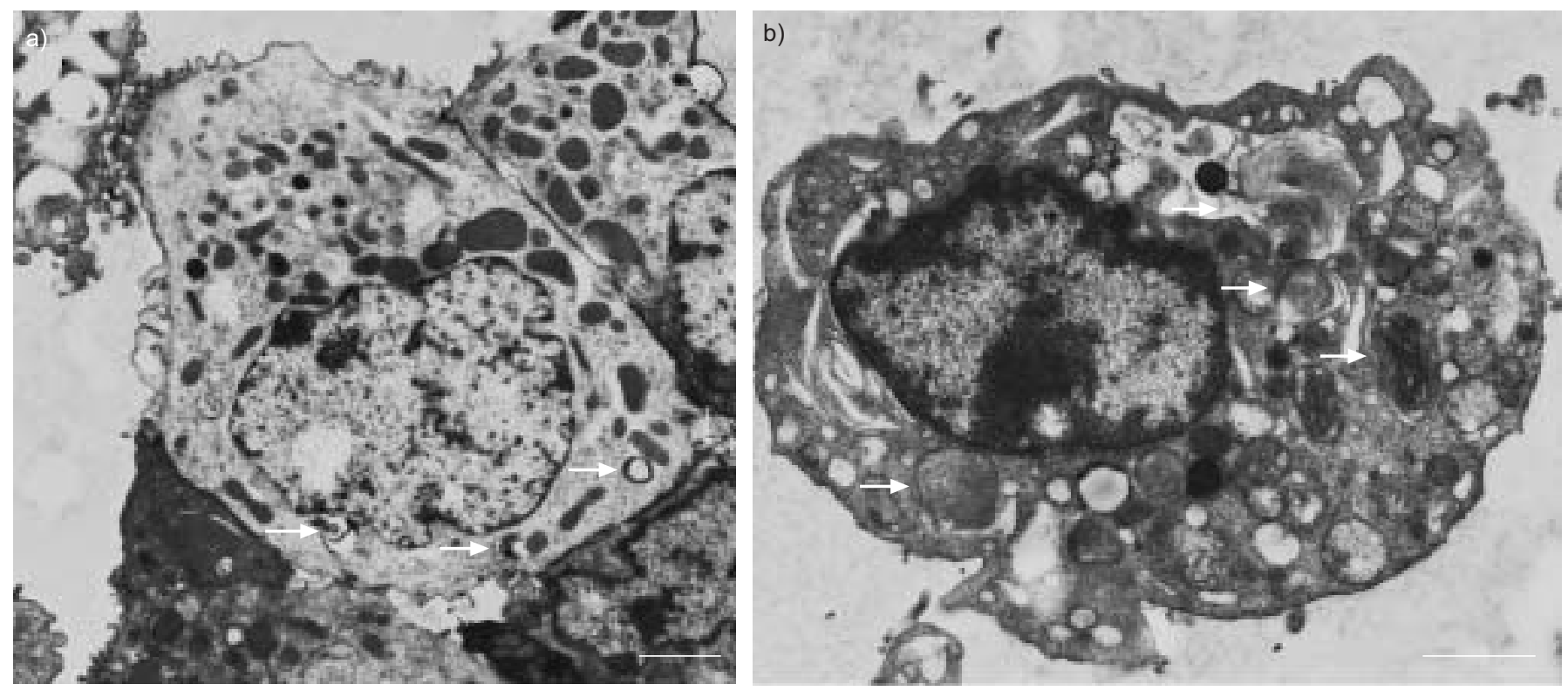

FIGURE 2. Electron micrographs of freshly purified peripheral lung epithelial cells. a) Clara cell with laminated inclusions (arrows) and a heterogeneous population of electron-dense granules. b) Type-II cell with characteristic lamellar bodies (arrows). Scale bars $=2 \mu \mathrm{m}$.

\section{E1A DNA, mRNA and protein in transfected cell lines}

E1A DNA was detected in E1A-PLEC clones 1, 2, 10 and 11 and E1A-NLF clones 1-4 (fig. 1a). Reverse transcriptase (RT)PCR of RNA from E1A-PLEC and E1A-NLF clones resulted in the amplification of two bands of the expected size from the $12 \mathrm{~S}$ and 13S E1A mRNAs, respectively, which were also present in RNA from A549 cells infected with adenovirus 5 (fig. 1h). No such bands were seen in RNA from primary NLFs, with RT-PCR of the 18S ribosomal RNA confirming equal RNA loading (data not shown). Immunohistochemistry showed strong nuclear staining for E1A in the E1A-PLEC and NLF transfected clones (fig. $1 \mathrm{~b}$ and c, respectively), whereas no staining was observed with isotype-matched control IgG (fig. 1d and e). On Western blots, the same two E1A monoclonal antibody-binding proteins were detected in E1APLECs and E1A-NLFs, and were similar in size to the two largest E1A proteins detected in G293 cells (American Type Culture Collection, Manassas, VA, USA; fig. 1f and g) [19]. The size markers indicate that these proteins have a relative molecular mass of $40-50 \mathrm{kDa}$, which is larger than that predicted from their amino acid sequence. Phosphorylation could account for part of this discrepancy, but, because of this discrepancy, it is difficult to assign the two to the $12 \mathrm{~S}$ and $13 \mathrm{~S}$ isoforms. Although mRNA abundance does not necessarily predict protein abundance, mRNAs representing both isoforms are expressed by these two cells (as previously discussed).

\section{Electron microscopy}

In a total of 324 cells from a freshly isolated PLEC preparation, $28 \%$ were alveolar macrophages, $25 \%$ exhibited features characteristic of Clara cells, including laminated vesicular inclusions and a heterogeneous population of electron-dense granules (fig. 2a), 25\% were type-II cells with characteristic lamellar bodies (fig. 2b), 12\% were alveolar type-I cells, $6 \%$ were ciliated bronchiolar epithelial cells and $4 \%$ were eosinophils. No fibroblasts were identified in these electron microscopic studies.
Primary PLECs grown on Matrigel-coated polycarbonate inserts formed three-dimensional (3D) aggregates composed of polarised cuboidal cells facing a central lumen, with numerous microvilli at the cell apices, lamellar inclusions in the cytoplasm, and intercellular junctional complexes (fig. 3a). In contrast, E1A-PLEC clones grown in the same manner formed 3D aggregates composed of three or four cuboidal cells with junctional complexes but lacking lamellar bodies or microvilli (fig. 3b). When grown on uncoated polycarbonate filters, these cells formed monolayers of polarised cuboidal cells with junctional complexes (fig. 3c).

NLFs grown on uncoated polycarbonate filters formed two types of clusters in the same dish. Some clusters were composed of monolayers of spindle-shaped cells with long cytoplasmic extensions (fig. 4a), and others of multiple layers of cells of irregular morphology (fig. $4 \mathrm{~b}$ ). No junctional complexes were observed in either type. E1A-NLF clones, conversely, formed monolayers of polarised cuboidal cells with junctional complexes (fig. $4 \mathrm{c}$ and d).

\section{Immunohistochemistry}

Table 1 summarises the immunohistochemical results shown in figure 5. Primary PLECs (fig. 5a), E1A-PLEC clones (fig. 5b) and E1A-NLF clones (fig. 5c) all showed cytoplasmic staining for cytokeratin, whereas primary NLFs did not (fig. 5d). Staining intensity was similar in E1A-PLEC and E1A-NLF clones, but significantly less than in primary PLECs (table 1).

All four cell types showed positive cytoplasmic staining for vimentin (fig. 5e-h), with no significant difference in staining intensity between the cells (table 1).

Primary PLECs showed weak perinuclear staining for smooth muscle $\alpha$-actin (fig. $5 \mathrm{i}$ ), whereas NLFs showed strong cytoplasmic staining (fig. 5l). In contrast, no staining was observed in E1A-PLEC (fig. 5j) or E1A-NLF clones (fig. 5k). 

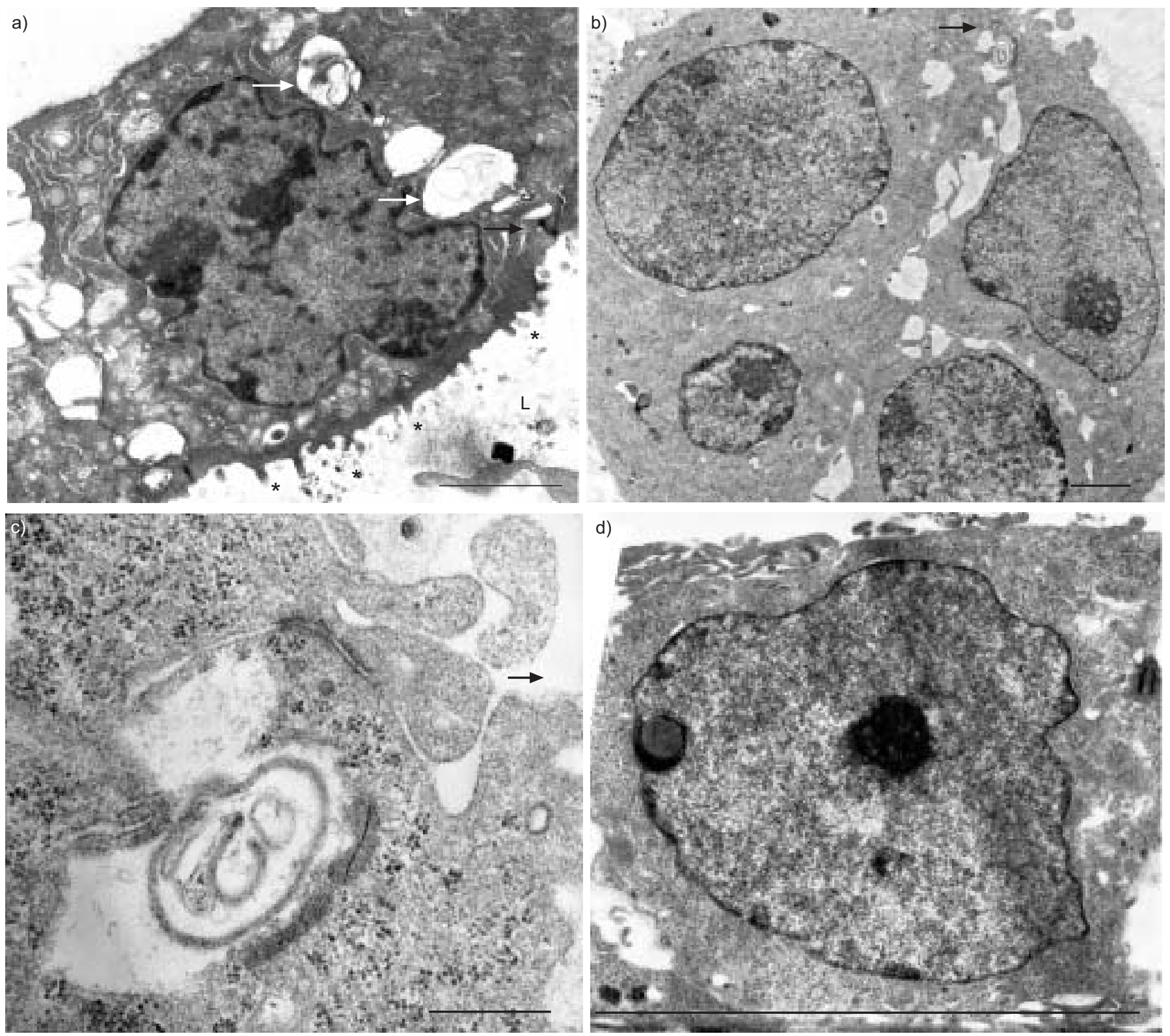

FIGURE 3. Electron micrographs of purified peripheral lung epithelial cells (PLECs) in primary culture and E1A-PLEC clone. a) Primary cultured PLECs grown on Matrigel-coated polycarbonate filter forming three-dimensional aggregates of cuboidal cells, with lamellar bodies (white arrows), junctional complexes (black arrow) and apical microvilli $\left(^{*}\right)$ lining a central lumen (L). b) E1A-PLEC clone 1 grown on Matrigel-coated polycarbonate filter with junctional complex (black arrow) between cells. c) Higher magnification of junctional complex shown in b). d) E1A-PLEC clone 1 grown on an uncoated polycarbonate filter forming a monolayer of cells with polarised cuboidal morphology and junctional complexes (arrow). Scale bars $=2 \mu \mathrm{m}$.

Contaminating lung fibroblasts found around PLECs in primary culture were negative for cytokeratin (fig. 5a), but stained strongly for vimentin (fig. 5e) and smooth muscle $\alpha$ actin (data not shown).

No staining was observed when cells were stained with isotype-matched control antibodies (fig. $5 \mathrm{~m}-\mathrm{p}$ ).

\section{Surfactant protein mRNA expression}

Northern hybridisation (fig. 6) showed that guinea pig lung and freshly purified PLECs (lanes 1 and 2, respectively) express surfactant protein A, B and C mRNAs. Primary cultures of PLECs grown on Matrigel-coated polycarbonate filters expressed only surfactant protein B mRNA (lane 3), and those grown on plastic dishes did not express surfactant protein mRNAs (lane 4). E1A-PLEC clones grown on either Matrigel-coated polycarbonate filters (lanes 5 and 6 ) or plastic dishes (data not shown) did not express surfactant protein mRNAs. In these cells, $18 \mathrm{~S}$ ribosomal RNA, used as an internal control, was similar (fig. 6d).

\section{EMSA}

LPS stimulation induced NF-kB binding activity in nuclear extracts of E1A-NLFs and E1A-PLECs but not NLFs (fig. 7a). In 

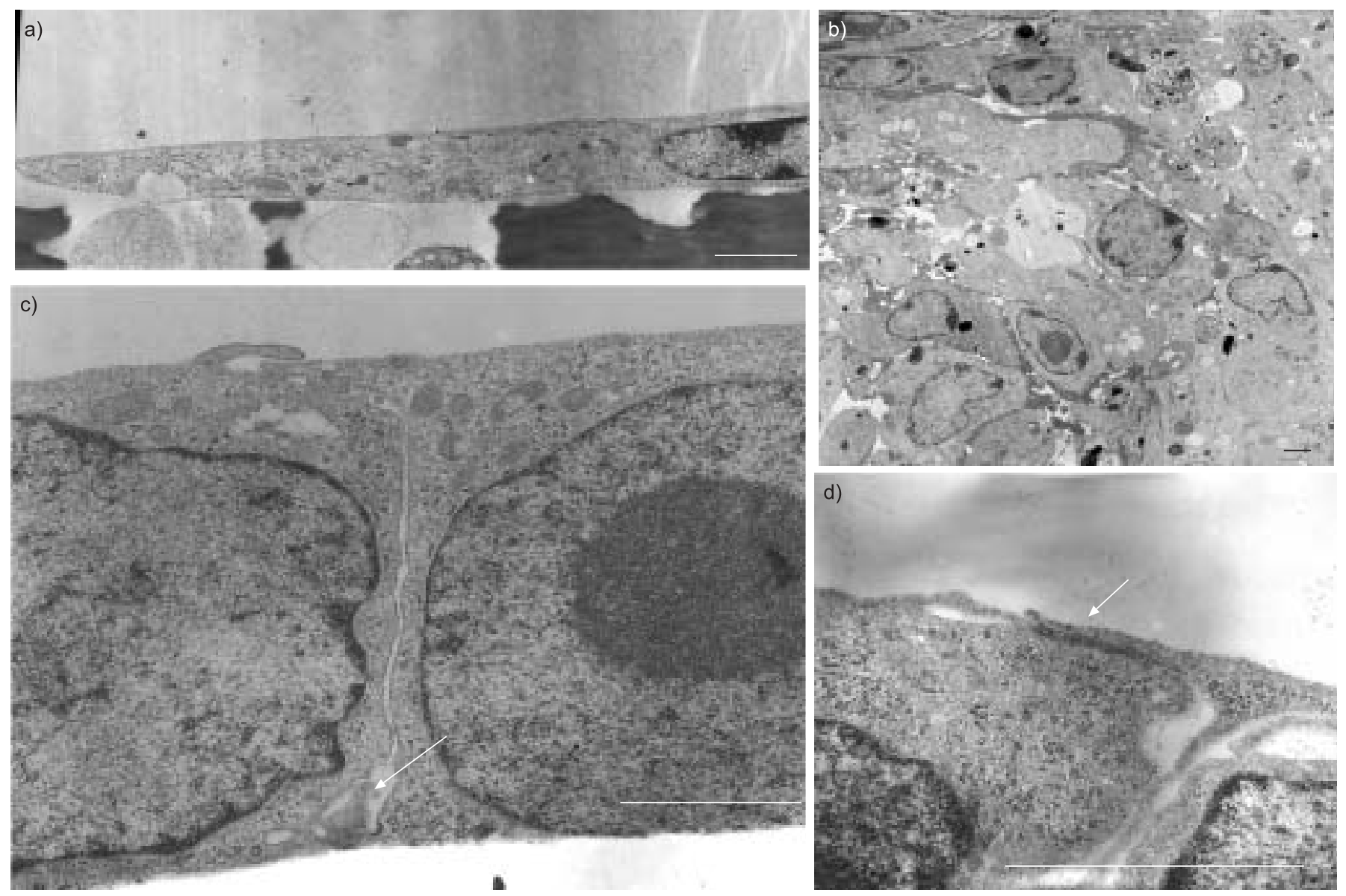

FIGURE 4. Electron micrographs of normal lung fibroblasts (NLFs) and E1A-NLF clone 1 grown on uncoated polycarbonate filters. NLFs form areas of a) monolayer and b) multilayer growth. c) E1A-NLF clone 1 grows as a monolayer of cells with cuboidal morphology and junctional complexes (arrow). d) Higher magnification of an apical junctional complex (arrow) between two adjacent E1A-NLF cells. Scale bars $=2 \mu \mathrm{m}$.

E1A-PLECs, the specific binding complexes detected were numbered I to III in order of their migration through the gel, from fastest to slowest, with complex II exhibiting the greatest intensity (lane 6). Binding activity in E1A-NLFs was represented by complexes that generally migrate faster than those in E1A-PLECs, with a possible overlap with complex I (lane 4) but a lower intensity of binding than found in E1A-PLECs.

EMSA of AP-1 demonstrated constitutive binding in nuclear extracts of NLFs that was not altered by LPS stimulation, whereas, in E1A-NLFs and E1A-PLECs, LPS increased AP-1 binding (fig. $7 \mathrm{~b}$ ). The bands representing AP-1 binding in E1ANLFs migrated faster than the single NLF or double E1A-PLEC bands.

In E1A-PLEC supershift assays, the use of antibodies directed against p50 and p65 for NF-אB and JunB, c-jun and c-fos for $\mathrm{AP}-1$ and competition assays using excess unlabelled specific or unrelated oligonucleotides confirmed the specificity of the proteins bound to the respective oligonucleotides (fig. $7 \mathrm{c}$ and d). With respect to NF- $\mathrm{kB}$, the antibody directed against p65 mainly supershifted complexes III and II, whereas the antibody directed against p50 shifted complexes I and II (fig. 7c). This suggests that p50 and p65 are part of complex II. With respect to AP-1, both c-Jun and c-Fos antibodies produced supershifted bands, whereas JunB antibodies did not (fig. 7d). The E1A-NLF supershifted bands paralleled those found in E1A-PLECs, for both NF-кB and AP-1 (data not shown), except the supershifted bands originated from the smaller complexes formed by these transcription factors in E1A-NLFs.

\section{DISCUSSION}

The present results show that adenovirus E1A DNA can be used to immortalise guinea pig PLECs and fibroblasts. Clonally derived E1A-expressing PLECs show a limited number of type-II cell phenotypes, but failed to show more specific markers of this differentiation, such as laminated vesicular inclusions and microvilli or expression of surfactant protein mRNAs. When grown on uncoated plastic dishes, they showed some epithelial characteristics, such as weak cytokeratin expression and cobblestone morphology, and, when grown on uncoated polycarbonate filters, they showed cuboidal morphology and junctional complexes. These features are all consistent with previous reports characterising E1Aimmortalised cell lines established from primary cultures of rat type-II pneumocytes grown on uncoated plastic [20-22]. These rat cells exhibited epithelial phenotypes of cytokeratin expression, junctional complexes and cuboidal morphology and also lacked the differentiation markers of type-II cells. Despite the 


\begin{tabular}{|c|c|c|c|c|c|}
\hline \multirow[t]{3}{*}{ TABLE 1} & \multicolumn{5}{|c|}{$\begin{array}{l}\text { Characteristics of peripheral lung epithelial cells (PLECs) and normal lung fibroblasts (NLFs) and their E1A } \\
\text { transfectants }\end{array}$} \\
\hline & \multirow{2}{*}{$\begin{array}{l}\text { Cuboidal } \\
\text { morphology }\end{array}$} & \multicolumn{3}{|c|}{ Immunostaining } & \multirow[t]{2}{*}{ SP mRNA } \\
\hline & & Cytokeratin & Vimentin & Smooth muscle $\alpha$-actin & \\
\hline PLECS & + & $H^{*, \#}$ & + & $t^{*, \#}$ & + \\
\hline NLFs & - & - & + & H & ND \\
\hline
\end{tabular}

present attempt to improve the retention of primary differentiation by culturing the primary PLECs on matrix, before and during the transfection procedure, the transformed cells failed to maintain the specific features of type-II cells.

With respect to fibroblasts, the present findings suggest that E1A causes a mesenchymal-to-epithelial transformation. Although NLFs grow as monolayers of cells with spindleshaped morphology interspersed with multilayered foci of cells of irregular shape to give the culture a peak-and-valley appearance, E1A-expressing NLFs switch this parent phenotype for that reminiscent of PLEC transfectants. Such epithelialisation, including cuboidal morphology, junctional complexes and cytokeratin production, was further supported by downregulation of smooth muscle $\alpha$-actin, a mesenchymal marker that was strongly positive in the parent NLFs. These results are supported by the report of FRISCH [23] of adenovirus E1A-induced mesenchymal-to-epithelial transformation in human tumour cells of mesenchymal origin. The E1Aexpressing tumour cells, like the present transfected NLFs, expressed junctional complexes and cytokeratin. FRISCH [23] suggested that conversion to an epithelial phenotype requires that mesenchymal transactivators, which repress epithelial gene transcription, are either not expressed or inactivated by E1A, particularly by the $12 \mathrm{~S}$ form of E1A, which could be expressed by the E1A-NLFs in the present study. Based on this hypothesis, the introduction of E1A into mesenchymal cells, in the present case, could inactivate these repressors and result in the induction of epithelial genes. Indirect evidence for the lack of such a potential repressor in the present transfected NLFs comes from the results of the EMSA analysis of AP-1, where, compared with NLFs, transfected NLFs showed an AP-1 complex of greater mobility, i.e. a smaller complex, suggesting the possible absence of a repressor that was part of the AP-1 complex in the untransfected NLFs.

ALCORN et al. [24] showed that fibroblasts are a major source of contamination in primary cultures of type-II cells. In the present case, spindle-shaped fibroblast-like cells that were negative for cytokeratin, but positive for vimentin and smooth muscle $\alpha$-actin, comprised 5 and $15 \%$ of the primary PLEC preparation after 3 and 4 days of culture, respectively. This three-fold increase in fibroblasts compared with PLECs, whose numbers remained virtually unchanged, is indicative of the higher mitotic activity of the contaminating fibroblasts. Consequently, the E1A-transfected cells initially assumed to be PLECs could have arisen from contaminating fibroblasts, with subsequent mesenchymal-to-epithelial transformation, as suggested by FRISCH [23].

Contrary to the previous explanation, evidence has been presented that strongly suggests that the two types of E1Atransfected cells are different. Initially, differences in the DNAbinding activity of the transcription factors NF- $\mathrm{BB}$ and AP-1 of NLFs, E1A-NLFs and E1A-PLECs and changes in this activity in response to LPS stimulation were presented. Nuclear extracts from NLFs have very weak NF- $\mathrm{KB}$ binding activity, and this basal activity is not increased upon stimulation with LPS. E1A-transfected NLFs also show low basal binding activity, but, in contrast to NLFs, E1A-transfected cells respond to LPS with increased binding. Transfected PLECs, likewise, exhibit low basal activity, and, like transfected NLFs, respond to LPS, but, in this case, more strongly. Besides this difference in response strength, additional complexes, larger in size than that in transfected NLFs, are present. With respect to AP-1, NLFs show constitutive binding activity that is not affected by LPS. Transfected NLFs not only show a lower basal AP-1 binding activity but also form a smaller complex that is now responsive to LPS. Transfected PLECs also respond to LPS, but the complexes formed more closely resemble that found in untransfected NLFs. Although E1A enhanced NF- $\kappa B$ and AP-1 binding activity in response to LPS in both NLFs and PLECs, the differences in the transcriptional complexes formed in each cell demonstrate that these cells are inherently different, despite other phenotypic similarities induced by E1A.

The active DNA-binding forms of both NF- $\mathrm{\kappa B}$ and AP-1 are dimers, composed of up to five members of the NF- $\kappa \mathrm{B} / \mathrm{Rel}$ family for NF- $\mathrm{B}$ [25], and, for AP-1, of members of the Fos and Jun families, consisting of five and three members, respectively [26]. In order to identify the subunits that comprise the complexes formed by the two transcription factors and thus determine the source of the difference between the complexes formed in the transfected PLECs and NLFs, the supershift assay was used. Interestingly, the present results showed that 

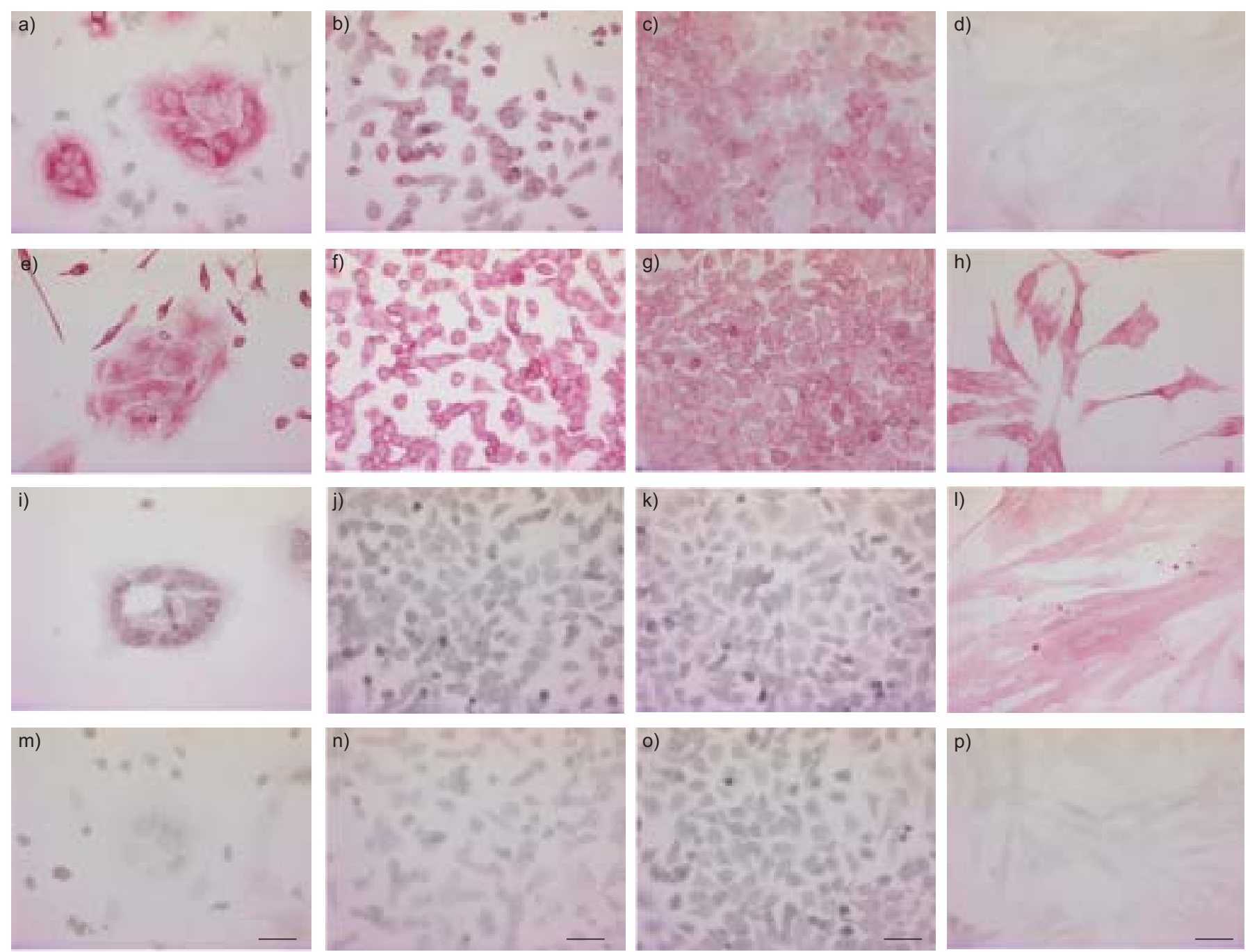

FIGURE 5. Immunostaining for a-d) cytokeratin, e-h) vimentin, $i-l)$ smooth muscle $\alpha$-actin and $m-p)$ controls. A primary peripheral lung epithelial cell (PLEC) culture (a, $\mathrm{e}, \mathrm{i}$ and $\mathrm{m}$ ), E1A-PLEC clone 1 (b, f, j and n), E1A-normal lung fibroblast (NLF) clone 1 (c, g, k and o) and NLFs ( $, \mathrm{h}, \mathrm{I}$ and p) were grown on coverslips and stained with either the specific monoclonal antibodies or isotype-matched immunoglobulin $\mathrm{G}$ (control). Contaminating fibroblasts are seen around epithelial colonies (a and e). Scale bars $=10 \mu \mathrm{m}$.

some of the subunits that contribute to the respective complexes in E1A-PLECs and E1A-NLFs are the same. Since the overall size of some of the binding complexes in the two cell types differs, this suggests that the other subunit forming the respective dimer might be different. In addition to this, or alternatively, proteins that associate with these transcription factors in the process of building the multiprotein transcriptional complex may exhibit cell type-specific expression [27] and thus contribute to the size differences in the sequencespecific DNA-binding complexes identified in the two cell types. These possibilities of cell type-specific expression of transcription factor subunits and/or associated factors further support the present authors' argument that E1A-transfected PLECs did not originate from transfection of contaminating fibroblasts. Therefore, it is concluded that transfection of PLECs and NLFs results in E1A-expressing cell lines with intermediate epithelial-mesenchymal features that may be related to dedifferentiation induced by E1A. This conclusion is in agreement with RAMON Y CAJAL et al. [28], who transformed epithelial and fibroblastic tumour cells with adenovirus E1A and found that both converged to similar phenotypes, regardless of cell origin.

E1A induced a more mesenchymal phenotype in guinea pig PLECs, i.e. loss of surfactant protein and reduction in cyokeratin expression, markers of epithelial cells, but retention of vimentin expression. Epithelial-mesenchymal transformation has been documented in fibroblasts originating from epithelial cells in progressive kidney disease [10], idiopathic pulmonary fibrosis [11] and liver cirrhosis, in which hepatic stellate cells converted to myofibroblasts [29]. Since adenovirus E1A inhibits the transcriptional activity of many promoters [30-32], E1A repression of differentiation markers in PLECs may induce a process whereby PLECs first lose their differentiation markers to move into an intermediate phenotype and then, possibly by transactivation of mesenchymal genes by its 135 isoform [33], reach the mesenchymal phenotype. 
a)

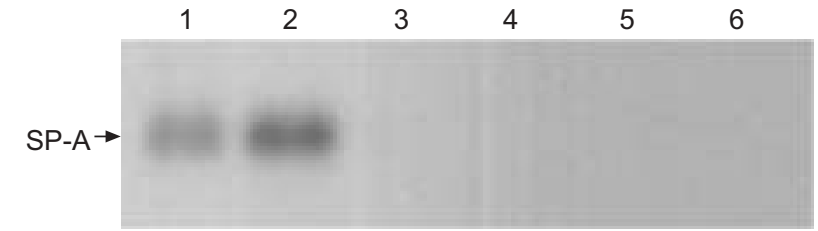

b)

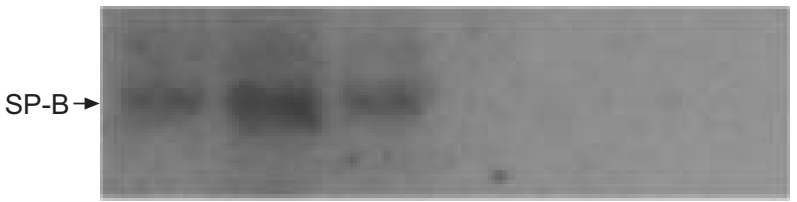

c)

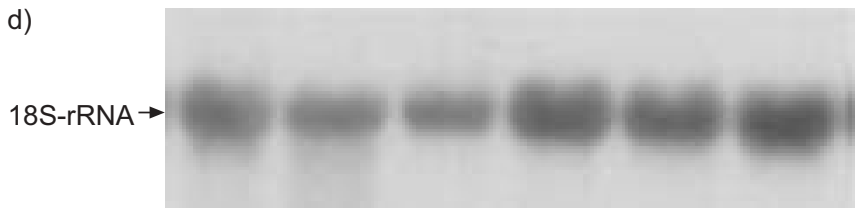

FIGURE 6. Northern blot analysis to determine expression of a) surfactant protein (SP)-A, b) SP-B and c) SP-C mRNA in total cellular RNA extracted from a guinea pig lung (lane 1), freshly purified peripheral lung epithelial cells (PLECs; lane 2), a primary culture of purified PLECs grown on a Matrigel-coated polycarbonate filter (lane 3), the same cells grown on plastic (lane 4), and E1A-PLEC clones 1 and 10 grown on a Matrigel-coated polycarbonate filter (lanes 5 and 6). d) $18 \mathrm{~S}$ ribosomal RNA (rRNA) was used as an internal control.

The epithelial-mesenchymal transformation induced by adenovirus E1A in guinea pig peripheral lung epithelial cells is particularly interesting since remodelling of the peripheral airways with increased thickening of their walls was recently shown to be associated with chronic obstructive pulmonary disease severity [2]. The present authors suspect that mesenchymal transformation of epithelial cells might contribute to the remodelling process observed in the peripheral lung in chronic obstructive pulmonary disease by deregulating matrix deposition in the airway wall. The E1A-induced transformation of peripheral lung epithelial cells, therefore, provides support for a role of adenovirus infection in the pathogenesis of the airway remodelling process. These guinea pig cells also provide a model for studying the underlying mechanisms leading to the expression of a more mesenchymal phenotype as well as related changes that may affect airways remodelling. Furthermore, the present model of persistent E1A expression after adenoviral infection in the guinea pig could provide a useful means of studying this aspect of the pathogenesis of chronic obstructive pulmonary disease in vivo as it can amplify the effects of chronic cigarette smoke exposure and increase polymorphonuclear leukocyte, macrophage and CD4 and CD8 lymphocyte numbers in the peripheral airways [8], something not apparently observed in mice. Also, this guinea pig model, in which only a minority of lung epithelial cells retain E1A [9], might better reflect the situation in human lungs [4] compared with a transgenic mouse model of lung-specific
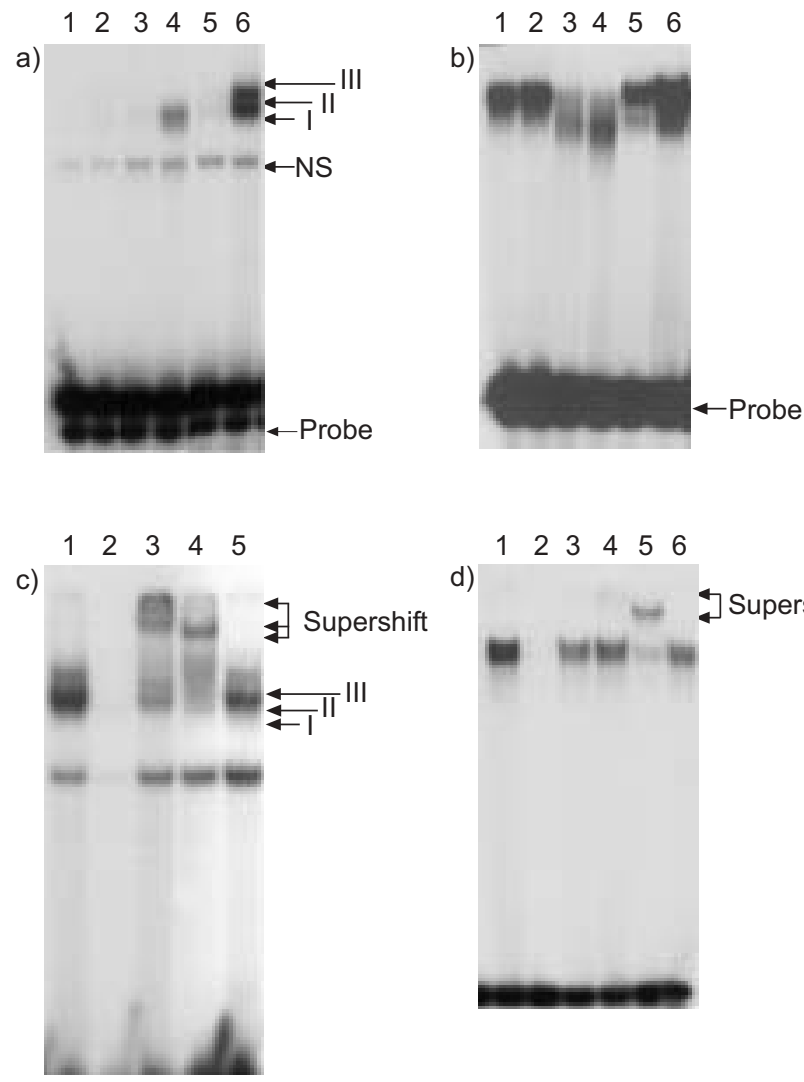

$123 \quad 3 \quad 4 \quad 56$

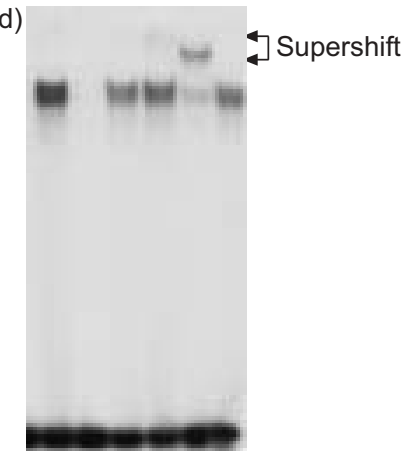

FIGURE 7. a, b) Electrophoretic mobility shift assay (EMSA) detection of nuclear factor (NF)- $\mathrm{kB}$ and activator protein (AP)-1 binding activity in normal lung fibroblasts (NLFs; lanes 1 and 2), E1A-NLF clone 1 (lanes 3 and 4) and E1Aperipheral lung epithelial cell (PLEC) clone 1 (lanes 5 and 6). The assay was performed on nuclear extracts from untreated cells (lanes 1, 3 and 5) and cells stimulated with $10 \mu \mathrm{g} \cdot \mathrm{mL}^{-1}$ lipopolysaccharide (LPS) for $2 \mathrm{~h}$ (lanes 2, 4 and 6) using a) the immunoglobulin ( $\mathrm{Ig}) \kappa \mathrm{NF}-\mathrm{KB}$ probe, 5'-AGTTGAGGGGACTTTCCCAGGC-3' (NF-KB binding sequence GGGACTTTCC) and b) the AP-1 probe, 5'CGCTTGATGAGTCAGCCGGAA-3' (AP-1 binding sequence TGAGTCA). The positions of specific protein-DNA complexes (I-III), nonspecific complex (NS) and unbound probe are shown. C) NF-kB binding in nuclear extracts from E1APLECs stimulated with $10 \mu \mathrm{g} \cdot \mathrm{mL}^{-1}$ LPS for $2 \mathrm{~h}$ in the presence of excess unlabelled AP-1 (lane 1) or NF-KB (lane 2), oligonucleotides and antibodies directed against p65 (lane 3) or p50 (lane 4), or control IgG (lane 5). Complexes I-III and bands supershifted by p65 and p50 antibodies are indicated. d) AP-1 binding in nuclear extracts from E1A-PLECs stimulated with $10 \mu \mathrm{g} \cdot \mathrm{mL}^{-1} \mathrm{LPS}$ for $2 \mathrm{~h}$ in the presence of excess unlabelled NF-KB (lane 1) or AP-1 (lane 2), oligonucleotides and antibodies directed against JunB (lane 3), c-Jun (lane 4), c-Fos (lane 5), or control IgG (lane 6). Bands supershifted by C-Jun and c-Fos antibodies are shown. Similar results were obtained for both NF-KB and AP-1 binding activity when a different pair of cells, namely E1A-NLF clone 2 and E1A-PLEC clone 2, were compared.

E1A expression [33], in which all alveolar and bronchiolar cells [34] have the potential to express these viral proteins.

\section{ACKNOWLEDGEMENTS}

The authors would like to thank F. Graham (McMaster University, Hamilton, ON, Canada) for the pE1Aneo plasmid, and D. English and the late S. Greene (both from James Hogg iCapture Center for Cardiovascular and Pulmonary Research, 
St Paul's Hospital, Vancouver, BC, Canada) for technical assistance with digital images.

\section{REFERENCES}

1 Pauwels RA, Buist AS, Calverley PM, Jenkins CR, Hurd SS. Global strategy for the diagnosis, management, and prevention of chronic obstructive pulmonary disease. NHLBI/WHO Global Initiative for Chronic Obstructive Lung Disease (GOLD) Workshop summary. Am J Respir Crit Care Med 2001; 163: 1256-1276.

2 Hogg JC, Chu F, Utokaparch S, et al. The nature of smallairway obstruction in chronic obstructive pulmonary disease. N Engl J Med 2004; 350: 2645-2653.

3 Fletcher CM, Peto R, Tinker CM, Speizer FE. The Natural History of Chronic Bronchitis and Emphysema. Oxford, Oxford University Press, 1976.

4 Elliott WM, Hayashi S, Hogg JC. Immunodetection of E1A proteins in human lung tissue. Am J Respir Cell Mol Biol 1995; 12: 642-648.

5 Matsuse T, Hayashi S, Kuwano K, Keunecke H, Jefferies WA, Hogg JC. Latent adenoviral infection in the pathogenesis of chronic airways obstruction. Am Rev Respir Dis 1992; 146: 177-184.

6 Retamales I, Elliott WM, Meshi B, et al. Amplification of inflammation in emphysema and its association with latent adenoviral infection. Am J Respir Crit Care Med 2001; 164: 469-473.

7 Vitalis TZ, Kern I, Croome A, Behzad H, Hayashi S, Hogg JC. The effect of latent adenovirus 5 infection on cigarette smoke-induced lung inflammation. Eur Respir J 1998; 11: 664-669.

8 Meshi B, Vitalis TZ, Ionescu D, et al. Emphysematous lung destruction by cigarette smoke. The effects of latent adenoviral infection on the lung inflammatory response. Am J Respir Cell Mol Biol 2002; 26: 52-57.

9 Behzad AR, Hayashi S, Hogg JC. Localization of latent adenovirus 5 E1A DNA in human lungs and lymph nodes by PCR in situ. Am J Respir Crit Care Med 1998; 157: A448.

10 Okada $H$, Inoue $T$, Suzuki $H$, Strutz $F$, Neilson EG. Epithelial-mesenchymal transformation of renal tubular epithelial cells in vitro and in vivo. Nephrol Dial Transplant 2000; 15: Suppl. 6, 44-46.

11 Chilosi M, Poletti V, Zamo A, et al. Aberrant Wnt/ $\beta$ catenin pathway activation in idiopathic pulmonary fibrosis. Am J Pathol 2003; 162: 1495-1502.

12 Dobbs LG, Gonzalez R, Williams MC. An improved method for isolating type II cells in high yield and purity. Am Rev Respir Dis 1986; 134: 141-145.

13 Morikawa O, Walker TA, Nielsen LD, Pan T, Cook JL, Mason RJ. Effect of adenovector-mediated gene transfer of keratinocyte growth factor on the proliferation of alveolar type II cells in vitro and in vivo. Am J Respir Cell Mol Biol 2000; 23: 626-635.

14 Shannon JM, Emrie PA, Fisher JH, Kuroki Y, Jennings SD, Mason RJ. Effect of a reconstituted basement membrane on expression of surfactant apoproteins in cultured adult rat alveolar type II cells. Am J Respir Cell Mol Biol 1990; 2: 183-192.
15 Pan T, Mason RJ, Westcott JY, Shannon JM. Rat alveolar type II cells inhibit lung fibroblast proliferation in vitro. Am J Respir Cell Mol Biol 2001; 25: 353-361.

16 Williams MC. Conversion of lamellar body membranes into tubular myelin in alveoli of fetal rat lungs. J Cell Biol 1977; 72: 260-277.

17 Cordell JL, Falini B, Erber WN, et al. Immunoenzymatic labeling of monoclonal antibodies using immune complexes of alkaline phosphatase and monoclonal antialkaline phosphatase (APAAP complexes). J Histochem Cytochem 1984; 32: 219-229.

18 Keicho N, Higashimoto Y, Bondy GP, Elliott WM, Hogg JC, Hayashi S. Endotoxin-specific NF- $\kappa B$ activation in pulmonary epithelial cells harboring adenovirus E1A. Am J Physiol Lung Cell Mol Physiol 1999; 277: L523-L532.

19 Whyte P, Ruley HE, Harlow E. Two regions of the adenovirus early region $1 \mathrm{~A}$ proteins are required for transformation. J Virol 1988; 62: 257-265.

20 Mallampali RK, Floerchinger CS, Hunninghake GW. Isolation and immortalization of rat pre-type II cell lines. In Vitro Cell Dev Biol 1992; 28A: 181-187.

21 Pasternack M, Floerchinger CS, Hunninghake GW. E1Ainduced immortalization of rat type II alveolar epithelial cells. Exp Lung Res 1996; 22: 525-539.

22 Steele MP, Levine RA, Joyce-Brady M, Brody JS. A rat alveolar type II cell line developed by adenovirus 12SE1A gene transfer. Am J Respir Cell Mol Biol 1992; 6: 50-56.

23 Frisch SM. E1a induces the expression of epithelial characteristics. J Cell Biol 1994; 127: 1085-1096.

24 Alcorn JL, Smith ME, Smith JF, Margraf LR, Mendelson CR. Primary cell culture of human type II pneumonocytes: maintenance of a differentiated phenotype and transfection with recombinant adenoviruses. Am J Respir Cell Mol Biol 1997; 17: 672-682.

25 Rothwarf DM, Karin M. The NF- $\mathrm{BB}$ activation pathway: a paradigm in information transfer from membrane to nucleus. Science STKE 1999; 5: re1-16.

26 Kaminska B, Pyrzynska B, Ciechomska I, Wisniewska M. Modulation of the composition of AP-1 complex and its impact on transcriptional activity. Acta Neurobiol Exp 2000; 60: 395-402.

27 Lemon B, Tjian R. Orchestrated response: a symphony of transcription factors for gene control. Genes Dev 2000; 14 : 2551-2562.

28 Ramon y Cajal S, Sanchez-Prieto R, Anaya A. A genetic model for undifferentiated cell tumor formation: similar tumors formed by different cell lines transformed by the E1A oncogene. Histol Histopathol 1995; 10: 811-820.

29 Bachem MG, Meyer D, Melchior R, Sell KM, Gressner AM. Activation of rat liver perisinusoidal lipocytes by transforming growth factors derived from myofibroblastlike cells. A potential mechanism of self perpetuation in liver fibrogenesis. J Clin Invest 1992; 89: 19-27.

30 Hen R, Borrelli E, Chambon P. Repression of the immunoglobulin heavy chain enhancer by the adenovirus2 E1A products. Science 1985; 230: 1391-1394.

31 Sogawa K, Handa H, Fujisawa-Sehara A, Hiromasa T, Yamane M, Fujii-Kuriyama Y. Repression of cytochrome 
P-450c gene expression by cotransfection with adenovirus E1a DNA. Eur J Biochem 1989; 181: 539-544.

32 Stein RW, Ziff EB. Repression of insulin gene expression by adenovirus type 5 E1a proteins. Mol Cell Biol 1987; 7: 1164-1170.

33 Yang Y, McKerlie C, Borenstein SH, et al. Transgenic expression in mouse lung reveals distinct biological roles for the adenovirus type 5 E1A 243- and 289-amino acid proteins. J Virol 2002; 76: 8910-8919.

34 Glasser SW, Korfhagen TR, Wert SE, et al. Genetic element from human surfactant protein SP-C gene confers bronchiolar-alveolar cell specificity in transgenic mice. Am J Physiol Lung Cell Mol Physiol 1991; 261: L349-L356. 